\title{
Ácidos graxos trans: doenças cardiovasculares e saúde materno-infantil ${ }^{1}$
}

\section{Trans fatty acids: cardiovascular diseases and mother-child health}

Vera Lucia CHIARA ${ }^{1,2}$

Rosilaine SILVA ${ }^{3}$

Renata JORGE ${ }^{3}$

Ana Paula BRASIL ${ }^{4}$

\section{R E S U M O}

Este estudo revê a literatura sobre o tema nas últimas décadas, destacando seu efeito no metabolismo humano quanto às doenças coronarianas e à saúde materno-infantil. Recentemente, os ácidos graxos trans foram incluídos entre os fatores dietéticos de risco para doenças cardiovasculares. Discute-se ainda sua relação com o processo de crescimento e desenvolvimento da criança desde a fase fetal e período gestacional. Os trans originam-se dos ácidos graxos insaturados no processo de hidrogenação e bio-hidrogenação, apresentando ação diferenciada destes. Diversas pesquisas ressaltam seu efeito hipercolesterolêmico e 0 bloqueio e inibição da biossíntese de ácidos graxos essenciais. Estas ações têm repercussões na saúde materno-infantil e elevam o risco de doenças cardiovasculares. Recomenda-se a redução do consumo de alimentos que contenham gordura hidrogenada, adotando os limites de $2 \%$ a $5 \%$ de gorduras trans/energia totais, já empregados em outros países.

Termos de indexação: ácidos graxos trans, doenças cardiovasculares, gravidez, saúde materno-infantil, consumo de alimentos.

\footnotetext{
1 Trabalho elaborado a partir da tese de doutorado em Epidemiologia de autoria de V.L. CHIARA, "Avaliação nutricional de adolescentes como instrumento na prevenção de doenças cardiovasculares". Instituto de Medicina Social, Universidade do Estado do Rio de Janeiro, 2000.

2 Instituto de N utrição, Universidade do Estado do Rio de Janeiro. Rua São Francisco Xavier n. 524, 12ํandar, sala 12001, Maracanã, 20559-900, Rio de Janeiro, RJ, Brasil. Correspondência para/Correspondenceto: V.L. CHIARA. E-mail: chiara@uerj.br ou vchiara@easynet.com.br

3 Bolsistas PIBIC/SR2/UERJ, Acadêmicas do Curso de Nutrição, Instituto de N utrição, Universidade do Estado do Rio de Janeiro.

${ }^{4}$ Nutricionista.
} 


\section{A B S T R A C T}

This article reviews the literature produced on the theme during the last decades, focusing on its effect on human metabolism, concerning coronary diseases and mother-child health. Trans fatty acids have been recently included among dietary risk factors for cardiovascular diseases. Their relation with the child's growing and developing processes, since fetal phase and gestational period, has also been discussed. Trans fatty acids originate from unsaturated fatty acids, in hydrogenation and biohydrogenation processes, and their action is different from the latter. Many works emphasize their hypercholesterolemic effect and influence on blocking and inhibiting biosynthesis of essential fatty acids. These actions reflect on mother-child health and increase the risk of cardiovascular diseases. The author recommends the consumption of food containing hydrogenated fat within the limits of $2-5 \%$ of trans fatty acids for total calories, as in other countries.

Index terms: fatty acids, trans, cardiovascular diseases, pregnancy, maternal and child health, food consumption.

\section{N T R O D U Ç Ã O}

Os efeitos adversos dos isômeros trans na saúde humana têm sido objeto de diversos estudos nas últimas décadas, associando-os às doenças cardiovasculares (DCV), ao processo de crescimento e desenvolvimento da criança e à fase gestacional.

As contradições nos resultados das pesquisas que os correlacionaram com as doenças cardiovasculares contribuíram para o surgimento, em 1995, de um consolidado do International Life Sciences Institute (International..., 1995), concluindo haver poucas evidências de associação entre o consumo de ácidos graxos trans e 0 aumento do risco destas doenças. No entanto, a Organização M undial da Saúde (OMS), ainda em 1995, posicionou-se reconhecendo a relação entre ácidos graxos trans e doenças coronarianas (World..., 1995), enquanto, em 1996, o mesmo International Life Sciences Institute, através da equipe Task Force on Trans Fatty Acids, baseada em diversos trabalhos apresentando associação entre trans e DCV, passou a reconhecer os trans como importante fator de risco para as DCV (American Society...,1996).

Estudos sobre a associação entre os ácidos graxos trans e a saúde materno-infantil ainda são escassos em humanos, predominando em animais. Todavia, algumas pesquisas levantam hipóteses sobre a possível relação, enquanto outros apontam efeitos deletérios sobre o processo de crescimento e desenvolvimento, além de interferência no período gestacional (Carlson et al., 1997).

No presente estudo, são revistas as publicações que abordam as características básicas dos ácidos graxos trans e sua ação no organismo humano em relação às doenças coronarianas e à saúde materno-infantil, observando-se ainda as recomendações de consumo adotadas em outros países.

Foram consultados a base de dados do Medline correspondente ao período 1980-2000 e anais de seminários e congressos durante a década de 90 .

\section{Características básicas dos ácidos graxos trans}

A maior parte dos ácidos graxos insaturados presentes nos alimentos existe na forma cis, significando que os hidrogênios estão do mesmo lado da dupla ligação. Osácidos graxos trans formados a partir dos insaturados apresentam inversão na dupla ligação, colocando o hidrogênio na posição transversal e provocando a linearização da cadeia (Harper, 1994).

Ao contrário dos isômeros cis, os trans são praticamente inexistentes em óleos e gorduras de origem vegetal não-refinados. No entanto, pequeninas porções de isômeros trans podem ser 
formadas durante reações químicas, como a oxidação que ocorre durante a extração, refinação e armazenamento dos óleos vegetais (Geuking, 1995).

Os ácidos graxos trans, também denominados gorduras trans, não são sintetizados no organismo humano e resultam de processo natural de bio-hidrogenação ou de processo industrial de hidrogenação parcial ou total de óleos vegetais ou marinhos. A bio-hidrogenação representa pequena contribuição adicional ao consumo e ocorre por digestão de gorduras ingeridas por animais ruminantes poligástricos, sendo conseqüente da ação de enzimas do rúmen (Geuking, 1995).

No processo de hidrogenação, os ácidos graxos trans originam-se da mistura de hidrogênio aos óleos insaturados, sob temperatura apropriada e com a presença de elemento catalisador o qual é posteriormente retirado. A quantidade de trans formada na hidrogenação pode ser controlada pelo catalisador e pela temperatura (Geuking, 1995). As finalidades desse processo são conferir ponto de fusão mais elevado aos óleos vegetais, prover maior estabilidade à oxidação lipídica e reduzir o tempo de cozimento (Okonek et al., 1996).

Os trans têm maior ponto de fusão que seus equivalentes cis. 0 ponto de fusão das gorduras insaturadas é sempre inferior ao das saturadas e trans (Aro et al., 1997). O ponto de fusão do ácido graxo oléico é de $13^{\circ} \mathrm{C}$, enquanto o de seu isômero trans, o ácido graxo elaídico, é de $44^{\circ} \mathrm{C}$. Essa diferença tem sido atribuída à orientação linear das moléculas nos isômeros trans (Jones \& Kubow, 2000).

Os ácidos graxos trans apresentam diversas configurações em virtude da localização onde ocorre a isomerização, podendo estar na forma trans-cis (t,c), cis-trans (c,t) ou trans-trans (t,t). A apresentação da posição dos diferentes tipos de isômeros na cadeia carbônica pode ser expressa colocando-se primeiramente o comprimento da cadeia de carbono, seguido das informações so bre a posição da dupla ligação e isomerização (C 18:2 9c 12t) (Greyt et al., 1996).
O metabolismo dos trans tem sido estudado e os resultados demonstram controvérsias quanto à sua semelhança com os ácidos graxos insaturados ou saturados. Debates ainda existem sobre as diferenças entre os trans originados de ácidos graxos monoinsaturados e poliinsaturados. No entanto, acredita-se que os trans, embora apresentem duplas ligações em suas estruturas como os insaturados, revelam metabolismo semelhante aos saturados (Booyens \& Merwe, 1992; Judd et al., 1994; Mensink \& Hornstra, 1995).

Os isômeros trans são digeridos, absorvidos e incorporados pelo organismo humano de forma similar aos ácidos graxos com isômeros cis, não apresentando, entretanto, atividade como ácidos graxos essenciais (Khosla \& Hayes, 1996).

Os isômeros cis são mais rapidamente metabolizados como fonte de energia que os trans, e são preferencialmente incorporados em fosfolipídios estruturais e funcionais. Em humanos a incorporação dos trans nos tecidos depende da quantidade ingerida, do tempo consumindo alimentos com esse tipo de gordura, da quantidade de ácidos graxos essenciais consumida, do tipo de tecido e do tipo de isômero (configuração e posição da dupla ligação na cadeia) (Geuking, 1995). Os teores encontrados em tecidos adiposos refletem o consumo por longo período de tempo, apresentando normalmente correlação com relato de ingestão por mais de um ano (Garland et al., 1998).

\section{Ácidos graxos trans e doenças coronarianas}

0 principal efeito metabólico dos ácidos graxos trans em relação às doenças coronarianas refere-se à sua ação hipercolesterolêmica, elevando o colesterol total e a lipoproteína de baixa densidade ( $L D L-C)$, reduzindo a lipoproteína de alta densidade (HDL-C) e resultando em significativo aumento na relação da LDL-c/HDL-C. Alguns autores consideram essa relação como 0 prognóstico mais importante para DCV quanto aos 
níveis de lipídios plasmáticos (Willett \& Ascherio, 1995).

Sugere-se que o efeito redutor dos trans sobre HDL-c se passe através da transferência de ésteres de colesterol de HDL-c para LDL-c (Khosla \& Hayes, 1996).

Uma das ações dos lipídios trans relacionadas ao aumento de LDL-c refere-se à supressão da atividade do LDL-receptor (Jones \& Kubol, 2000). As LDL-c são resultantes do catabolismo de lipoproteínas de muito baixa densidade (VLDL) que contêm apolipoproteína b (apo-b). Através da apo-b o LDL-receptor capta a LDL-c, removendo-a da circulação via hepática. Variações mínimas de LDL-receptor no fígado afetam os níveis plasmáticos de LDL-c. Assim, quando os níveis do LDL-receptor estão baixos por ação de nutrientes ou efeito genético, irá ocorrer maior acúmulo de LDL-c no plasma, elevando o risco de doença arterial coronariana (DAC) (Semenkovich, 2000). Lipídios da dieta podem também afetar a secreção de apo-b, contribuindo para o surgimento de hiperlipidemia (Aro et al., 1997).

Acredita-se que após os 20 anos 0 mecanismo de regulação de LDL-receptor diminui sua eficiência, afetando os níveis de LDL-c plasmáticos. No entanto, esse processo provavelmente não progride após os 50 anos. Isto sugere que, após esta fase, os fatores externos podem desempenhar importante função para 0 controle dos níveis séricos de colesterol. A atividade de LDL-receptor também diminui em mulheres após a menopausa, em conseqüência da queda de produção de estrogênio (Grundy, 2000).

Outro aspecto relevante quanto ao efeito desse tipo de gordura sobre os níveis de LDL-C refere-se à ação competitiva entre os ácidos graxos trans e os poliinsaturados. Estes atuam elevando o número de receptores de LDL-c e aqueles interferem no metabolismo de ácidos graxos poliinsaturados, atuando sobre a enzima dessaturase, a qual age no metabolismo do ácidos graxos essenciais, constituindo-se em bloqueadores e inibidores na síntese dos ácidos graxos essenciais de cadeia longa (Dupont, 1991).

Dietas ricas em competidores e moderadores de ácidos graxos essenciais podem produzir mudanças na produção e formação de prostaglandinas e tromboxanos (eicosanóides), que têm como precursores os ácidos graxos poliinsaturados com cadeias acima de 20 carbonos. Esses efeitos não acontecem se a quantidade de ácidos graxos essenciais for ingerida adequadamente. A ação dos trans sobre os poliinsaturados também ocorre por substituição destes nos tecidos (Jones \& Kubow, 2000).

Os eicosanóides modulam a função de muitas células cardiovasculares. Os tromboxanos facilitam a agregação plaquetária, enquanto as prostaglandinas atuam inversamente. 0 balanço entre tromboxanos e prostaglandinas tem sido considerado importante para a adequada função cardiovascular. $\mathrm{Na}$ aterosclerose, doença cardiovascular com maior ocorrência em todo 0 mundo, ocorre a formação de placa fibrogordurosa (ateroma), resultante de agregação plaquetária (Harper, 1994).

Alguns estudos levantaram a hipótese de que os trans elevam o nível de lipoproteína (a), que é af etada por poucos componentes da dieta, além de contribuir para o aumento dos triglicerídeos. Todavia, desconhecem-se os processos metabólicos causadores de tais efeitos (Katan, 1998).

\section{Ácidos graxos trans e saúde materno-infantil}

Diversos estudos vêm abordando a relação entre consumo de ácidos graxos e a fase gestacional, sugerindo-se que os trans são transferidos ao feto através da placenta. Para Honstra (2000), deve haver prudência quanto a considerar-se a associação entre estes fatores, pois as evidências ainda são insuficientes. Koletzko e Müller (1990) encontraram teores de trans no plasma materno diretamente proporcionais aos do 
cordão umbilical. Essa quantidade elevada de trans, quando comparada aos teores encontrados em outros tecidos, pode ser conseqüente do tipo de tecido do cordão umbilical, que apresenta maior concentração de lipídios, levando à maior incorporação de trans.

Entre as pesquisas voltadas para a análise da ação dos isômeros trans sobre a saúde da criança, encontrou-se como relato comum o bloqueio e inibição na biossíntese dos ácidos graxos poliinsaturados de cadeia longa, na fase fetal e após o nascimento (Koletzko, 1992). 0 estudo de Koletzko \& M üller (1990) demonstrou correlação inversamente proporcional e significativa $(r=-0,47$ e $p<0,01$ ) entre ácido graxo linoléico e trans. Acredita-se que esse processo ocorra através da inibição da enzima dessaturase (Carlson et al., 1997).

Sugere-se, ainda, que os trans afetam o crescimento intra-uterino por inibição da biossíntese dos ácidos graxos poliinsaturados araquidônico e docohexaenóico (Decsi \& Koletzko, 1995). A hipótese para explicar o efeito dostrans sobre o retardo do crescimento se sustenta no importante papel desempenhado pelos ácidos graxos essenciais, especialmente 0 docohexaenóico, no processo de crescimento, associando-se positivamente com o peso e comprimento de nascimento e circunferência do crânio (Crawford, 2000). Além disso, observou-se correlação inversa entre consumo de trans e o peso ao nascer $(r=-0,50$ e $p<0,01)$ (Koletzko, 1995).

A credita-se que as gorduras trans podem afetar também o processo de desenvolvimento da criança. Esse fenômeno também foi explicado pela deficiência na formação dos mesmos ácidos araquidônico e docohexaenóico, os quais estão envolvidos na função psicomotora (Booyens \& Merwe, 1992).

Níveis de trans em fosfolipídios plasmáticos foram associados inversamente com o período de tempo da gestação $(r=-0,17$ e $p<0,001)$, sugerindo-se ser esta relação inversa decorrente de redução na síntese do ácido docohexaenóico.
Foi observada ainda associação inversa entre níveis de trans plasmáticos em ésteres de colesterol de mulheres com filhos nascidos à termo e outras com filhos pré-termo $(r=-0,59$ e $p<0,005)$ (Jendryczko et al., 1997).

Outro efeito verificado entre os ácidos graxos trans e a gestação refere-se ao aumento do risco de pré-eclâmpsia. Analisando a pressão arterial em dois grupos de mulheres, estudiosos observaram que aquelas relatando consumo mais elevado de gorduras trans durante a gestação apresentaram maior risco de manifestação desta complicação. Os resultados do estudo mostraram níveis médios de trans superiores em $28 \%$ para as mulheres com pré-eclampsia, sendo a diferença estatisticamente significante $(p<0,001)$. Os autores relataram chances maiores de complicações entre as mulheres com os mais elevados níveis de trans, sendo 0 odds ratio de 7,4 com intervalo de confiança de 1,4 a 39,7 (95\% ), mesmo depois de ajustado o modelo por diversas variáveis de confundimento. A pesquisa concluiu que o risco de pré-eclampsia parece elevar-se de forma linear com o aumento dos níveis de trans $(p=0,05)$. A hipótese metabólica para explicar esse processo ainda está sob investigação em estudo de caráter longitudinal (Williams et al., 1998).

Considera-se que o consumo de trans no período gestacional pode contribuir para que o processo de aterogênese tenha início ainda na fase intra-uterina. A ação dos trans na deficiência de ácido linoléico propiciaria alteração na divisão das células musculares lisas da íntima (Booyens \& M erwe, 1992).

Os trans maternos podem ser transferidos para a criança também através da amamentação. Análise do leite materno em mulheres francesas encontrou níveis médios de trans de 1,9 $\pm 0,2 \%$ do total de lipídios (Chardigny et al., 1995), detectando-se, ainda, teores plasmáticos de trans em crianças americanas com aleitamento materno exclusivo (Innis \& King, 1999). 0 teor de trans do leite materno apresenta alta correlação com a quantidade ingerida, seja referente ao consumo atual ou de longo prazo $(r=0,909$ e $p<0,05)$ (Craig 
et al., 1984). A partir dos teores de ácidos graxos trans do leite humano aferidos em estudo desenvolvido no Canadá, estimou-se o consumo de $3,7 \mathrm{~g}$ a $10,6 \mathrm{~g} /$ pessoa/dia em mulheres lactantes. Concentrações diferenciadas de trans foram detectadas entre o colostro e o leite subseqüente, considerando-se a quantidade consumida (Ratnayake $\&$ Chen, 1996).

\section{Recomendações de consumo}

Os ácidos graxos trans são encontrados em gorduras vegetais hidrogenadas, margarinas sólidas ou cremosas, cremes vegetais, biscoitos, sorvetes, pães, batatas fritas, pastelarias, bolos, tortas, massas, entre outros alimentos. A manufatura desses produtos e o conseqüente consumo têm se elevado ao longo dos anos no Brasil. As margarinas vêm substituindo a manteiga no consumo habitual do brasileiro nas últimas quatro décadas (M ondini \& M onteiro, 1995). No entanto, comparadas a similares estrangeiros, as margarinas e creme vegetais nacionais apresentam elevados teores de gorduras trans (Soares \& Franco, 1990). Atualmente, observa-se que o mercado produtor do país já vem oferecendo margarina cujo processo de hidrogenação foi substituído por interesterificação, levando à redução dos teores de gorduras trans no produto (Geuking, 1995).

A Organização Mundial da Saúde (W orld..., 1995) preconiza o controle no consumo de alimentos que contenham ácidos graxos trans, com vistas à prevenção e tratamento de doenças coronarianas. Entretanto, o valor quantitativo desse consumo não foi determinado. Por outro lado, também são desconhecidos os teores de gorduras trans nos alimentos, em face da ausência de informações em seus rótulos e em tabelas de composição química, dificultando o estabelecimento de recomendações quantitativas quanto à ingestão. Além disso, são raros os estudos analisando estes teores em alimentos consumidos no país (Chiara, 2000). Recentemente, a Food and Drug Administration (Food..., 1999) sugeriu a discriminação dos teores de gorduras trans em rótulos dos produtos, recomendando que, caso seja computado no conteúdo das gorduras saturadas, deve ser demarcado por símbolo informativo a quantidade específica de trans. Da mesma forma, sugere o Órgão que a ausência de trans deve também constar dos rótulos.

No Brasil, desde 1997, as gorduras trans deixaram de ser computadas como insaturadas, sendo incluídas no total de saturadas (Brasil, 1997). Recentemente, vem sendo revista pelo M inistério da Saúde a normatização dos rótulos, no sentido de oferecer ao consumidor informações básicase essenciais para o conhecimento dos nutrientes componentes do produto.

Alguns países, como França, Canadá, Inglaterra, Dinamarca, Nova Zelândia e outros, têm recomendado valores de consumo de $2 \%$ a $5 \%$ de gordura trans em relação à ingestão total de energia diária (Bolton et al., 1995; Nelson, 1998).

Quanto à saúde da gestante e da nutriz, foi sugerida por Carlson et al. (1997) a necessidade de equilíbrio entre o consumo de ácido graxo linoléico e trans, além da garantia de suprimento adequado de todos os ácido graxos essenciais na dieta. Os autores defendem que o consumo de trans seria de $10 \%$ no máximo, em relação a energia total da dieta diária materna. Para Carrol (1989), não deve haver gordura trans em qualquer tipo de produto industrializado voltado para alimentação infantil.

\section{O N C LUSÃ O}

Conforme sugerem os estudos analisados, as gorduras trans podem desempenhar importante efeito deletério na saúde humana. Sua ação quanto às DCV vem sendo amplamente reconhecida e divulgada. Embora existam diversas recomendações sobre o controle do seu consumo, a ausência de informações em tabelas de composição química de alimentos e em rótulos dos produtos consumidos no país prejudica essa ação. 
Com base na literatura consultada, considera-se que orientações para redução e controle de consumo de produtos com gordura hidrogenada devem ser incentivadas junto à população, especialmente em grupos de risco para patologias reconhecidamente associadas à ingestão de gorduras trans. 0 incentivo ao hábito da leitura de rótulos de produtos seria outro aspecto positivo para identificar os ácidos graxos trans, através da presença de gordura hidrogenada.

Por outro lado, o reduzido número de pesquisas demonstrando a ação deletéria das gorduras trans sobre a saúde materno-infantil impossibilitam a implementação de recomendações efetivas sobre o consumo neste grupo populacional. Todavia, o reconhecimento dos seus malefícios, quando comparadas às gorduras saturadas - anteriormente consideradas o principal fator de risco dietético para doenças coronarianas - é importante neste contexto, pois se sabe da possibilidade de início do processo aterogênico ainda na fase fetal. Assim, o controle da ingestão de ácidos graxos trans no grupo materno-infantil também parece oportuno. Outro aspecto relevante seria o efeito competitivo entre as gorduras trans e as essenciais para a saúde humana, especialmente neste segmento populacional. Garantir o consumo das gorduras essenciais é, mais do que nunca, prioritário para a saúde.

Deve-se levar em consideração, ainda, a crescente oferta de alimentos manufaturados no mercado consumidor do país, sabendo-se que muitos destes apresentam elevados teores de gorduras hidrogenadas em sua composição. Medidas visando à completa informação em rótulos de produtos podem ser muito úteis, apontando-se também campanhas publicitárias junto à população para estimular o hábito de leitura dos rótulos, bem como o incentivo à produção de alimentos com reduzidos teores de gorduras trans.

\section{REFERÊ NCIAS B I B LIO G RÁ F I C A S}

ARO, A., JAUHIAINEN, M ., PARTANEN, R., SALM INEN, I., MUTANEN, M. Stearic acid, trans fatty acids, and dairy fat: effects on serum and lipoprotein lipids, apolipoproteins, lipoprotein(a), and lipid transfer proteins in healthy subjects. American J ournal of Clinical Nutrition, Bethesda, v. 65, n.5, p.1419-1426, 1997.

AMERICAN SOCIETY FOR CLINICAL NUTRITION. Task Force on Trans fatty acids. Position paper on trans fatty acids. American Journal of Clinical Nutrition, Bethesda, v.63, n.4, p.663-670, 1996.

BOLTON, C.S., WOODWARD, M., FENTON, S. MCCLUSEY, M.K., BROWN, C.A. Trans fatty acids in the Scottish diet: an assessment using a semi-quantitative food-frequency questionnaire. British Journal of Nutrition, London, v.74, p.661-670, 1995.

BOOYENS, J., MERWE, van der C.F. Margarines and coronary artery disease. M edicals Hypotheses, v.37, p.241-244, 1992.

BRASIL. M inistério da Saúde. Secretaria de Vigilância Sanitária. Portaria no 521, de 17/10/97. Dispõe sobre Food Base. Informação Nutricional Complementar (521-97). Edição atualizada e revisada até setembro de 1997. Brasília, 1997. CD-ROM.

CARLSON, S., THOM AS, M.C., COOK, H.W., EM KEN, E.A., FILER Jr., L. Trans FATTY acids: infant and fetal development. American Journal of Clinical Nutrition, Bethesda, Bethesda, v.66, n.3, p.717S-736S, 1997. Supplement.

CARROL, K.K. Upper limits of nutrients in infant formulas polyunsaturated fatty acids and trans fatty acids. Journal of Nutrition, Bethesda, v.119, n.12, p.1810S-1813S, 1989. Supplement.

CHARDIGNY, J.M., WOLFF, R.L., M AGER, E., SEBEDIO, J.L., MARTINE, L., JUANEDA, P. Trans mono- and polyunsaturated fatty acids in human milk. European Journal of Clinical Nutrition, London, v.49, n.7, p.523-531, 1995. 
CHIARA, V.L. Avaliação nutricional de adolescente como instrumento de prevenção de doenças coronarianas. Rio de Janeiro: 2000. 91p. Tese (Doutorado em Saúde Coletiva/Epidemiologia) - Instituto de Medicina Social, Universidade Estadual do Rio de Janeiro, 2000.

CRAIG, M.C.S., WEETE, J.D., FAIRCLOTH, S.A., WICKWIRE, M.A., LIVANT, E.J. The effect of hydrogenated fat in the diet of nursing mothers on lipid composition and prostaglandin content of human milk. American Journal of Clinical Nutrition, Bethesda, v.39, n.6, p.778-786, 1984.

CRAWFORD, M.A. Placental delivery of arachidonic and docosahexaenoic acids: implications for the lipid nutrition of preterm infants $1,2,3$. American Journal of Clinical Nutrition, Bethesda, v.71, n.1, p.275-284, 2000.

DECSI, T., KOLETZKO, B. Do trans fatty acids impair linoleic acid metabolism in children? Annais Nutrition and Metabolism, v.39, n.1, p.36-41, 1995.

DUPONT, J. Lípidos. In: ORGANIZACIÓN Panamericana de la Salud. Conocimientos actuales sobre nutrición. 6.ed. Washington DC, 1991. p.66-77.

GARLAND, M., SACKS, F.M., COLDITZ, G.A., RIMM , E.B., SAMPSON, L.A., WILLETT, W.C. The relation between dietary intake and adipose tissue composition of selected fatty acids in US women. American Journal of Clinical Nutrition, Bethesda, v.67, n.1, p.25-30, 1998.

GEUKING, W. Factors determining trans isomerization in partial hidrogenation of edible oils. In: CONGRESO Y EXPOSICIÓN LATINOAM ERICANOS SOBRE PROCESSAM IENTO DE GRASAS Y ACETES, 6., 1995, Campinas. Anais... Campinas, 1995. p.139-142.

GREYT, W., KELLENS, M., HUYGHEBAERT, A. Contribution of trans fatty acids from vegetable oils and margarines to the Belgian diet. Fatty/Lipid, v.98, n.1, p.30-33, 1996.

GRUNDY, S.M. Nutrition and diet in the management of hyperlipidemia and atherosclerosis. In: SHILS, M.E. Modern nutrition in health and disease. Part C. Prevention and management of cardiovascular disorders. 9.ed. Philadelphia : Lippincott Willians and Wilkins, 2000. p.1199-1214.

FOOD AND DRUG ADMINISTRATION. FDA proposes new rules for trans fatty acids in nutrition labeling, nutrient content clains, and health clains. Available from: <www.access.gpo.gov/su_docs>. Cited: 29 out. 1999.

HARPER, H.A. Manual de química fisiológica. 7.ed. São Paulo : Atheneu, 1994.

HONSTRA, G. Essential fatty acids in mothers and their neonates ${ }^{1,2,3,4}$. American Journal of Clinical Nutrition, Bethesda, v.71, n.5, p.1262S-1269S, 2000. Supplement.

INNIS, S.M., KING, D.J. Trans fatty acids in human milk are inversely associated with concentrations of essential all-cis n- 6 and n-3 fatty acids and determine trans, but not $n-6$ and $n-3$, fatty acids in plasma lipids of breast-fed infants ${ }^{1,2,3}$. American Journal of Clinical Nutrition, Bethesda, v.70, n.3, p.383-390, 1999.

INTERNATIONAL LIFE SCIENCES INSTITUTE. Trans fatty acids and coronary heart disease risk. Report of the expert. Panel on trans fatty acids and coronary heart disease. American Journal of Clinical Nutrition, Bethesda, v.62, n.3, p.518S-526S, 1995. Supplement.

JENDRYCZKO, A., GRUSZCZYNSKI, J., TOMALA, J., SZPYRKA, G. Unsaturated fatty acids of trans isomers in plasma of pregnant women and birth weight. Ginekologia Polska, v.64, n.3, p.113-116, 1997.

JONES, P.J.H., KUBOW, S. Lipids, Sterols, and Their Metabolites. In: SHILS, M.E. Modern nutrition in health and disease. Part A. Major dietary constituents and energy needs. 9.ed. Philadelphia : Lippincott Willians and Wilkins, 2000. p.67-93.

JUDD, J.T., CLEVIDENCE, B.A., MUESING, R.A., WITTES, J., SUNKIN, M .E., PODCZASY, J.J. Dietary trans fatty acids: effects on plasma lipids and lipoproteim of healthy men and women. American Journal of Clinical Nutrition, Bethesda, v.59, n.4, p.861-868, 1994. 
KATAN, M.B. Health effects of trans fatty acids. European Journal of Clinical Investigation, Bethesda, Oxford, v.28, n.4, p.257-258, 1998.

KHOSLA, P., HAYES, K.C. Dietary transmonounsaturated fatty acids negatively impact plasma lipids in humans: critical review of the evidence. Journal American College Nutrition, v.15, n.4, p.325-339, 1996.

KOLETZKO, B., M ÜLLER, J. Cis- and trans- fatty acids in plasma lipids of newborn infants and their mothers. Biology of the Neonate, Basel, v.57, n.3/4, p.172-178, 1990.

KOLETZKO, B. Trans fatty acids may impair biosynthesis of long-chain polyunsaturates and growth in man. Acta Paedriatric, n.81, n.4, p.302-306, 1992.

KOLETZKO, B. Potential adverse effects of trans fatty acids in infants and children. European Journal Medical Research, v.17, n.1, p.123-125, 1995.

MENSINK, R., HORNSTRA, G. The proportion of trans monounsatured fatty acids in serum triacylglycerols or platelet phospholipids as an objective indicator of their short-term intake in healthy men. British Journal of Nutrition, London, v.73, n.4, p.605-612, 1995.

M ONDINI, L., MONTEIRO, C.A. Mudanças no padrão de alimentação. In: MONTEIRO, C.A. (Org.). Velhos e novos males da saúde no Brasil: a evolução do país e de suas doenças. São Paulo : Hucitec, 1995. p.79-92.

NELSON, G.J. Dietary fat, trans fatty, and risk of coronary heart disease. Nutrition Reviews, New York, v.56, n.1, p.250-252, 1998.
OKONEK, D.V., BERBEN, P.H., MARTELLI, G. Precious metal catalysis for fats and oils applications. In: SEM INÁRIO DA SOCIEDADE BRASILEIRA DE ÓLEOS E GORDURAS, 1996, Campinas. Anais... São Paulo : Instituto Adolfo Lutz, 1996. p.39-46.

RATNAYAKE, W.M.N., CHEN, Z.Y. Trans, n-3, and n-6 fatty acids in Canadian Human Milk. Lipids, Champaign IL, v.31, p.S-279-S-282, 1996.

SEM ENKOVICH, C.F. Nutrient and genetic regulation of lipoprotein metabolism. In: SHILS, M.E. Modern nutrition in health and disease. Part C. Prevention and management of cardiovascular disorders. 9.ed. Philadelphia : Lippincott Willians and Wilkins, 2000. p.1191-1197.

SOARES, L.M.V., FRANCO, M.R.B. Níveis de trans isômeros e composição de ácidos graxos de margarinas nacionais e produtos hidrogenados semelhantes. Ciência e Tecnologia dos Alimentos, v.10, n.1, p.57-71, 1990.

WILLIAM S, M.A., KING, I.B., SORENSEN, T.K., ZINGHEIM, R.W., TROYER, B.L., ZEBELMAN, A.M. Risk of preeclampsia in relation to elaidic acids (trans fatty acid) in maternal erythrocytes. Ginecologic and Obstetric Investigation, Basel, v.46, n.2, p.84-87, 1998.

WORLD HEALTH ORGANIZATION. Nutrition. Science - Policy. WHO and FAO Joint Consultation: fats and oils in human nutrition. Nutrition Reviews, New York, v.53, n.7, p.202-205, 1995.

WILLETT, W.C., ASCHERIO, A. Health effects of trans fatty acids. American Journal of Clinical Nutrition, Bethesda, v.66, n.4, p.1006S-1010S, 1995. Supplement.

Recebido para publicação em 4 de maio e aceito 4 de outubro de 2001. 